\title{
Treatment of Adolescent Substance Use Disorders
}

\author{
Katherine A. Belendiuk, PhD* \\ Paula Riggs, $M D^{*}$
}

\author{
Address \\ * Department of Psychiatry, University of Colorado School of Medicine, 12469 \\ E. 17th Place, Mail Stop F478, Aurora, C0 80045, USA \\ Email: kat.belendiuk@ucdenver.edu \\ Email: paula.riggs@ucdenver.edu \\ Published online: 12 April 2014 \\ (C) Springer International Publishing AG 2014
}

Keywords Adolescence $\cdot$ Substance abuse $\cdot$ Substance dependence · Treatment - Substance use disorders · SUD

\section{Opinion statement}

Significant progress has been made in development and dissemination of evidence-based behavioral interventions for adolescents with substance use disorders (SUD). Medications have also shown promise in reducing substance use when used in conjunction with psychosocial treatment for adolescents with SUD, even in the context of co-occurring psychopathology. Although the efficacy or "probable efficacy" of the behavioral interventions discussed in this review have been established based on at least two randomized controlled trials, they produce relatively low abstinence rates and modest reductions in substance use that attenuate over time. Research has shown that abstinence rates may increase with the addition of abstinence-based incentives; however, post-treatment relapse rates remain high, with few treated adolescents sustaining abstinence one year post-treatment. This may be due to the paucity of continuing care, or post-treatment recovery support services, and the lack of integrated or concurrent treatment for co-occurring psychiatric conditions that contribute to poorer treatment outcomes. Thus, despite significant progress, there is clearly room for the improvement of existing treatment for adolescents with SUD. There is also a critical need to increase the availability of, and access to, substance and behavioral health treatment services for adolescents. Although $10-15 \%$ of U.S. high school students would currently meet diagnostic criteria for at least one SUD, only $10 \%$ of those who could benefit from substance treatment receive it. Five-year trends, showing significant increases in the use of marijuana and nonmedical prescription drugs among U. S. high school students, are evidence of the shortcomings of existing school-based interventions, and poor access to community-based substance treatment for non-juvenile-justice involved youth. There is clearly a need to adapt or develop more effective prevention, early interventions, and treatment for youth who are "at risk," as well as the increasing number of adolescents who have progressed to more serious substance involvement.

\section{Introduction}

Research in the past decade has led to a deeper understanding of the biological and environmental factors that increase the risk of adolescent-onset substance use disorders (SUD). Epidemiologic studies have 
shown that substance use disorders are among the most common mental health disorders in adolescents and young adults [1]. By the twelfth grade, at least half of American high school students have used an illicit drug, and $40 \%$ report past year use [2]. Of particular concern are five-year trends showing significant increases in marijuana and prescription drugs use. Regular and daily marijuana use is currently at 30-year peak levels with almost a quarter of high school seniors reporting using at least once per month [2]. This raises significant public health concerns in the light of recent research which shows regular marijuana use during adolescent development to be associated with a reduc-

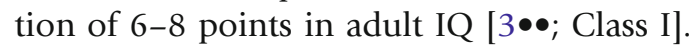

Fortunately, research has also led to the development of a number of effective behavioral interventions for substance abusing adolescents. Although behavioral interventions are considered to be "first line" treatment, medications are often used adjunctively to reduce drug cravings, symptoms of withdrawal, or to treat co-occurring psychiatric conditions. Although relatively few medication trials have been conducted in adolescents with SUD, several studies conducted in the past decade suggest that a handful of medications are relatively safe, well-tolerated, and may be helpful in the treatment of adolescents with SUD, based on the results of at least one randomized controlled trial. Specifically:-

1) Nicotine replacement therapy (NRT) and bupropion (alone or in combination) have been shown to increase quit attempts and smoking abstinence in nicotine-dependent adolescents [4];

2) N-acetylcysteine (NAC) has been shown to reduce marijuana craving and use in cannabis-dependent adolescents [5];
3) Six months of buprenorphine-naloxone treatment increased treatment compliance and retention, and reduced illicit and injection opioid use in opioid-dependent adolescents and young adults compared to those who received a brief 2-week detox taper of buprenorphine-naloxone [6];

4a) fluoxetine, for co-occurring major depressive disorder [7], and;

4b) osmotic-release methylphenidate [8•; Class I] and atomoxetine [9] for co-occurring ADHD have been shown to be relatively safe, well-tolerated, and promising for treating co-occurring psychiatric disorders in non-abstinent adolescents who are concurrently receiving outpatient substance treatment.

There is clearly a need for additional research to develop more effective substance treatment interventions and to increase access and availability to those interventions for youth and families. However, it is important to highlight that the effectiveness and costeffectiveness of addiction treatment has been shown to be comparable to treatments for other chronic medical illnesses such as hypertension, diabetes, and asthma $[10,11]$. It has been estimated that every US $\$ 1$ invested in addiction treatment yields a cost-saving of between US $\$ 4$ and US $\$ 7$ in reductions in drug-related crime, theft, and criminal justice costs. Savings can exceed costs by a ratio of 12 to 1 when societal and health-care related costs are considered, including increased workplace productivity, fewer drug-related accidents, reductions in addiction-related medical illnesses, and medical complications including overdoses and deaths [11].

\section{Treatment}

\section{Psychosocial/behavioral interventions}

- Behavioral or psychosocial interventions are considered "first-line" treatment for adolescents with SUD. The individual, group, and familybased interventions included in this review are limited to those with substantial empirical support for their efficacy, based on the results of at least two randomized controlled trials. 
- Overall, these interventions have been shown to have comparable, moderate effect sizes. Decisions regarding treatment matching should consider age, gender, co-occurring psychopathology, severity of substance use, family, psychosocial, and legal factors as potential moderators of treatment response [12]. There is some evidence that family-based interventions may be more appropriate for younger adolescents [12]. However, there is substantial empirical support for the efficacy of both group and individual CBT for adolescents ages 13-19. Moreover, CBT has consistently shown greater sustained or emerging post-treatment effect size compared to family-based interventions in adolescents $[13,14]$. There is also some evidence that individual CBT targeting SUD may contribute to reductions in psychiatric symptom severity in adolescents who have co-occurring psychiatric disorders such as depression [15], ADHD [8•, 9], and conduct disorder with depression [7].

Standard procedure

Contraindications

Complications

Special points

Cost/cost-effectiveness
There is substantial empirical support for the efficacy or "probable efficacy" of the following family-based adolescent substance treatment interventions: Multidimensional Family Therapy (MDFT) [1618]; Functional Family Therapy (FFT) [13]; Multisystemic Family Therapy (MST) [19]; Brief Strategic Family Therapy (BSFT) [20•; Class I]; and Adolescent Community Reinforcement Approach (ACRA) [13]. These interventions vary as to the number of adolescent-only, parent-only, and parent/adolescent family sessions, but all focus on improving: 1) adolescent functioning in family and social contexts; 2) parental monitoring skills and functioning in other adult roles; 3) communication between family and social systems (e.g., schools); and 4) improving adolescents' coping, communication, decision-making, and problem-solving skills associated with drug use.

Interventions include approximately 12-18 sessions (60-90 minutes) delivered at least weekly in a variety of settings (e.g., home, community, school, or clinic) over 4-5 months [11, 21].

Adolescents without family members who are willing to participate in treatment may receive greater benefit from group or individual treatment approaches.

None identified.

A-CRA provides significantly more individual CBT sessions with the adolescent-alone compared to other family-based interventions and has been successfully used with homeless and minority youth $[22,23]$.

The cost of family-based interventions can vary widely, ranging from approximately US $\$ 1500$ to US $\$ 9000$ per completed treatment episode. Rigorous, comparative cost-effectiveness studies in adolescents are lack- 
ing. A-CRA is generally at the lower end, and MST, MDFT, and FFT at the higher end, of the cost range [24, 25].
Standard procedure

Contraindications

Complications

Special points

Cost/cost-effectiveness
Both group and individual CBT have been shown to be efficacious for the treatment of substance use disorders and associated problems in adolescents $[18,26]$, with an average effect size in the moderate range $(\mathrm{d}=0.45)[27]$.

CBT focuses on enhancing adolescent coping, problem solving, and decision-making skills related to drug use; teaching skills to help adolescents cope with cravings and overcome temptations to use drugs (e.g., drug refusal skills, avoiding high risk situations); improving interpersonal relationships (e.g., communication, anger management, and mood regulation skills); and reducing risky behaviors associated with drug use (e.g., HIV/sexual risk behaviors, riding with or driving while intoxicated). Module selection can be tailored based on a functional analysis of an adolescent's triggers and contextual factors associated with drug use [11, 28, 29; Class 1]. Group-format CBT (e.g., MET/5; MET/12) may include one or two individual sessions to enhance their motivation to engage in treatment, before entering 3-10 weeks of weekly group CBT [24].

CBT can be modified for adolescents with more limited cognitive abilities, but may not be suitable for adolescents with significant cognitive impairment, or those with serious mood or psychotic symptoms. Serious exacerbations of co-occurring psychiatric conditions may need to be clinically addressed and managed before initiating CBT.

None identified.

Individual CBT may be preferred for adolescents with psychiatric comorbidity to better understand how psychiatric symptoms such as depression, anxiety, inattention, or frustration intolerance may trigger substance use.

The cost of CBT, with or without MET, can range from approximately US $\$ 900$ to US $\$ 4,000$ per completed treatment episode depending on the number of sessions/length of treatment, and whether delivered in group or individual format. Group CBT is generally less costly compared to individual CBT, but not necessarily more cost-effective [24].

Adjunctive and brief interventions- motivational enhancement

Motivational interviewing (MI) or motivational enhancement therapy (MET) is a non-judgmental, supportive, and non-confrontational style of interviewing [30]. Although motivational interviewing is often fully integrated throughout standard CBT substance treatment interventions, $\mathrm{MI} / \mathrm{MET}$ can also be used as an effective brief single-session, stand-alone intervention for adolescents who present or screen positive for problematic substance use or substance-related medical problems in medical office-based or hospital settings (e.g., emergency departments) [31]. Brief MI/MET interventions (1-3 sessions) are also used on the "front end" of longer substance treatment modalities such as CBT, to enhance patients' 
Standard procedure

Contraindications

Complications

Special points

Cost/cost-effectiveness self-efficacy and readiness for change, and motivation to engage in treatment [11]. At least four recent meta-analytic reviews show that $\mathrm{MI}$ is effective at reducing adolescent smoking $[32,33]$ and illicit drug use

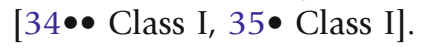

When used as a brief intervention MI/MET provides personal feedback, based on an initial clinical assessment, in an effort to increase patient awareness and insight regarding problems stemming from their substance use, with the goal of addressing ambivalence and eliciting internally-motivated commitment to changing substance use.

None identified.

None identified.

Brief MI/MET interventions from a physician/clinician may be effective for some patients to achieve sustained abstinence or reduce their substance use to non-problem levels. However, adolescents with more serious or chronic substance involvement generally require additional empirically-supported treatment following brief MI/MET interventions.

Comparing standard care (cost=US \$81) to a brief single session hospital emergency room MI/MET intervention (cost=US $\$ 170$, including screening), cost-effectiveness ratios favored MI/MET over standard care across all study outcomes. The societal cost savings of MI per quality-adjusted life year of were reported as US $\$ 8,795$ [31].

Adjunctive and brief interventions- contingency management and motivational incentives

Contingency management $(\mathrm{CM}) /$ motivational incentives are based on operant behavioral principles in which immediate rewards or incentives (i.e., voucher payments or prize drawings) are provided to increase the frequency of reinforced behaviors (e.g., abstinence, treatment compliance). $\mathrm{CM} /$ motivational incentives are generally used in conjunction with CBT or other behavioral substance treatment interventions, and have been shown to:-

1) produce greater reductions in drug/alcohol use, higher rates of abstinence, and longer periods of sustained abstinence compared to CBT alone [36, 37];

2) increase treatment compliance [38];

3) increase the frequency of involvement in non-drug, pro-social, or goal-related adolescent activities, after completing residential substance treatment.

$\mathrm{CM}$ /incentives have also been shown to increase smoking quit attempts and higher rates of smoking abstinence when used in conjunction with bupropion SR, compared to bupropion SR alone [4].

Standard procedure $\mathrm{CM}$ /incentives, in the form of voucher payments or prize drawings, are used to provide an immediate reward (i.e. behavioral reinforcement) when a targeted behavior is demonstrated (e.g., abstinence as verified by a negative urine drug screen). $\mathrm{CM}$ /incentives are also used to reinforce treatment 
Contraindications Behaviors that cannot be objectively measured are not appropriate for $\mathrm{CM}$ / incentives.

Complications Some institutions have accounting policies that pose barriers to implementing a CM program.

Special points Although CM involves chance with regard to prize draws, this intervention has not been shown to promote gambling behavior in adults [39]; to date no studies on CM's influence on gambling have been conducted in adolescents.

Cost/cost-effectiveness

The cost of $\mathrm{CM} /$ motivational incentives varies based on the magnitude and frequency of rewards provided for specified behaviors. The "fishbowl" method of intermittent reinforcement with opportunities to draw for small, medium, and large prizes was developed by Petry and colleagues [40] as a lower-cost alternative to voucher payment incentives. $\mathrm{CM} /$ incentives as low as US $\$ 0.30$ per patient per day increase the number of negative urine drug screens for opioids and cocaine and increase the proportion of negative urine drug screens for other drugs [41]. Billing related to increased patient retention from CM may exceed the cost of CM implementation.

Pharmacologic treatment

- Compared to a large body of research in adults, few pharmacotherapy trials have been conducted in adolescents with SUD [42].

- Buprenorphine-naloxone [6] and N-acetlycysteine [5] have been shown to be relatively safe and effective for reducing craving and use of opioids and cannabis, respectively, based on single placebo-controlled, randomized trials in adolescents who were concurrently enrolled/participating in psychosocial treatment for SUD.

- Fluoxetine for co-occurring major depressive disorder [7], osmotic-release methylphenidate (OROS-MPH) [8•], and atomoxetine for co-occurring ADHD [9] have been shown to be relatively safe, well-tolerated, and efficacious or "probably efficacious" for the treatment of a co-occurring psychiatric disorder in adolescents with SUD, based on single placebocontrolled randomized trials that included weekly, individual CBT.

\section{Substance use disorders}

- Buprenorphine is a partial agonist that can be delivered in a variety of clinical settings. It does not provide the euphoria and sedation caused by other opioids, reduces withdrawal symptoms, has a low risk of overdose, and is approved for treatment of individuals aged 16 and older. Buprenorphine can be administered sublingually as a monotherapy or combined with naloxone, an opioid antagonist in a 4:1 ratio. 
Standard dosage Up-titration:

Twelve weeks of buprenorphine-naloxone maintenance therapy has been shown to increase treatment retention and decrease opioid positive urines compared to two weeks of buprenorphine detoxification in a multi-site trial of adolescents and young adults [6]. Discontinuation of buprenorphine/naloxone maintenance was associated with relapse to opioid use at follow up for $48 \%$ of those treated.

Day 1: $2 \mathrm{mg}$ buprenorphine and $0.5 \mathrm{mg}$ naloxone with an additional 2 to $6 \mathrm{mg}$ of buprenorphine if needed after 1.5 to 2 hour observation

Days 2 and 3: Dose from prior day unless over- or under-medicated by clinical assessment with 2 to $6 \mathrm{mg}$ buprenorphine adjustment after 1.5 to 2 hour observation

Day 4: Dose from day 1 unless over- or under-medicated by clinical assessment with 2 to $6 \mathrm{mg}$ buprenorphine adjustment after 1.5 to 2 hour observation

Maximum dosing: $24 \mathrm{mg}$ per day with taper beginning at 9 weeks for 12 weeks of treatment Missed days: If a patient missed 3 consecutive days of treatment but returned prior to 7 days after the last dose, patients were given half the amount of the last dose and monitored for 1.5 hours. If the medication was tolerated then the patient was administered a portion or the remainder of the last dose.

Contraindications Patient requires chronic opioid analgesia.

Main drug interactions Antihistamines, conivaptan, dabrafenib, potassium salts, sodium oxybate.

Main side effects No serious adverse events were reported. Headaches were the most common side effect and less than $10 \%$ of the treatment population reported nausea, insomnia, stomach ache, vomiting, and anxiety. Constipation also common.

Special points Buprenorphine is a schedule III drug and restricted distribution in the USA requires special training by providers.

Cost/cost-effectiveness US $\$ 312.64$ for a 30 day supply.

- N-acetylcysteine (NAC) is an antioxidant that is widely available as an over-the-counter supplement. An eight-week randomized placebocontrolled trial comparing twice-daily administrations of NAC and placebo, in the context of a contingency-management intervention and brief weekly cessation counseling, demonstrated that adolescents administered NAC were 2.4 times more likely to have a negative urine cannabinoid test result during treatment compared to adolescents administered placebo [5].

Standard dosage $1200 \mathrm{mg}$ bid.

Contraindications None documented. 
Main drug interactions

Main side effects

Special points

Cost/cost-effectiveness
Nitroglycerin, activated charcoal may prevent NAC absorption when used to treat poisoning.

Upper respiratory infection, vivid dreams, insomnia, irritability, and heartburn.

NAC is available over-the-counter without a prescription.

US $\$ 15$ to US $\$ 20$ for 30 days of $1200 \mathrm{mg}$ bid.

\section{Treatment of co-occurring psychiatric disorders}

- $60 \%$ of adolescents with a substance use disorder also have at least one co-occurring mental disorder [43]. Therefore, screening and interventions should also address psychiatric comorbidity.

Fluoxetine for co-occurring major depressive disorder (MDD)

- Comorbid depression is associated with more severe substance dependence and higher rates of relapse. A 16-week randomized control trial of fluoxetine and CBT reduced symptoms on the Childhood Depression Rating Scale-Revised, self-reported substance use, and symptoms of conduct disorder [7].

Standard dosage

$20 \mathrm{mg}$ fixed daily.

Contraindications

None documented.

Main drug interactions

Isocarboxazid, linezolid, methylene blue, phenelzine, pimozide, procarbazine, selegiline transdermal, thioridazine, tranylcypromine.

Main side effects

Rates of adverse events were generally mild and transient.

Special points

Four patients in the fluoxetine group were evaluated in an emergency department or hospitalized due to concerns of worsening suicidality. Each patient endorsed psychosocial stressors precipitating increased suicidality, however the possibility that some adolescents may experience increased suicidality while being treated with fluoxetine cannot be excluded.

Cost/cost-effectiveness US $\$ 266.55$ for 10020 mg capsules.

Osmotic-release methylphenidate (OROS-MPH)

for co-occurring ADHD

- Individuals with ADHD have up to twice the risk of having a substance use disorder in their lifetime, compared to individuals without ADHD [44].

- 30-50 \% of adolescents with SUD have co-occurring ADHD.

- A multisite randomized control trial found that there were no differences in OROS-MPH and CBT compared to placebo and CBT for reductions in primary outcomes (i.e., reduction of ADHD symptoms and 
Standard dosage

Contraindications

Main drug interactions

Main side effects

Special points

Cost/cost-effectiveness days of substance use), but secondary outcomes (i.e., reductions in parent-rated ADHD symptoms at four and eight weeks, negative urine drug screens) favored OROS-MPH and CBT to placebo and CBT [8 ].

Participants were started on an $18 \mathrm{mg}$ dose and titrated to $72 \mathrm{mg}$ (or the highest dose tolerated) during the first two weeks of treatment.

MAO inhibitor within 14 days, severe cardiovascular disease, severe arrhythmias, motor tics, Tourette's syndrome.

Benzphetamine, diethylpropion, isocarboxazid, phendimetrazine, phenelzine, phentermine, procarbazine, selegiline transdermal, sibutramine, tranylcypromine.

Single study-related adverse event occurred, which was hospitalization for psychosis after ingestion of an unknown substance at a "rave."

No significant group differences between OROS-MPH and placebo on medication abuse or diversion.

US $\$ 657.86$ for $10036 \mathrm{mg}$ tablets
Standard dosage

Contraindications

Main drug interactions

Main side effects

Special points

Cost/cost-effectiveness
A randomized control trial found no differences between atomoxetine and MI/CBT, compared to placebo and MI/CBT for reductions in primary outcomes (i.e., reductions in ADHD symptoms, days of non-nicotine substance use) [9].

Participants were instructed to take the medication once daily in the morning. Those weighing less than $70 \mathrm{~kg}$ started at $0.5 \mathrm{mg} / \mathrm{kg}$ to $0.75 \mathrm{mg} / \mathrm{kg}$ per day and increased by $25 \mathrm{mg}$ per week until total dose was between 1.1 and $1.5 \mathrm{mg} / \mathrm{kg}$. Those weighing more than $70 \mathrm{~kg}$ started at $50 \mathrm{mg}$ per day, increased to $75 \mathrm{mg}$ per day in the second week and $100 \mathrm{mg}$ per day in the third week. Participants experiencing side effects remained at their current dose with titration occurring the following week.

MAO inhibitor within 14 days, glaucoma, cardiomyopathy, arrhythmias, cardiovascular disease, cardiac structural abnormalities, cerebrovascular disease.

Isocarboxazid, phenelzine, procarbazine, selegiline transdermal, tranylcypromine.

Rates of adverse events were generally mild and transient. One serious adverse event occurred for an atomoxetine-treatment patient when they had a seizure after taking an overdose of bupropion to hallucinate.

None identified.

US $\$ 286.92$ for 30 days of $100 \mathrm{mg}$ capsules

- Addiction is a complex, neurobiologically-based medical illness. As is true for other chronic diseases, including hypertension, asthma, and 
diabetes, changes in diet, exercise, or lifestyle can significantly impact mortality, morbidity, and prognosis [10]. Ongoing clinical management and recovery support from family, peers, or community-based recovery support services are important for maintaining optimal health and to prevent relapses or exacerbations of the illness [10].

- Engagement in community-based recovery support services such as 12-step programs (e.g., AA, NA) or other community-based selfhelp groups that emphasize reciprocal recovery support may be helpful to some adolescents $[45,46]$, but there is limited empirical support for 12-step programs with regard to improving long-term adolescent outcomes. However, there is growing empirical support for "assertive continuing care," a program that utilizes assertive linkage rather than passive referral to community-based recovery support services [47]. The program emphasizes continuity of contact and service in a primary recovery support relationship over time, especially during the vulnerable 90-day period after treatment. Trained recovery coaches or volunteer recovery support specialists contact patients and families to provide post-treatment monitoring and recovery support services, to facilitate the transition from recovery initiation to stable recovery maintenance.

\section{Emerging therapies}

\section{Telemedicine}

- There has been an increased interest in computer-assisted and telephonic treatments, particularly to increase access in rural and underserved areas [48].

- Telemedicine may be most applicable for an adolescent demographic because of the prevalence of technology in adolescent social connections and daily life [49] and high technological literacy.

- Tele-health is a flexible intervention that is available in numerous modalities (e.g., computer, text-messaging, video-messaging) and settings (e.g. home, school, work, emergency rooms, and health care providers' offices), allowing "on-demand" access to therapeutic support during times of risk for relapse [50].

Usage The addition of bi-weekly computer-based CBT to standard drug counseling increases the number of drug-free urine drug screens and duration of abstinence, with treatment benefits at six-month follow-up [51] and an internet-based intervention reduced alcohol, marijuana, and polydrug use at six-months post-treatment compared to controls [52]. Adolescent marijuana users randomized to a computer-based treatment reported fewer cannabis-related problems and lower use of other drugs that were in the small to medium effect size [53]. Text-messaging interventions aimed at promoting self-efficacy and skills use during selfreported triggering situations reduced marijuana use and desire in a triggering situation [54]. 
Special points Anonymity afforded by technology may facilitate treatment for individuals who are sensitive to disclosing sensitive information like substance use [55]. Systems need to be developed to address issues of privacy, payment, jurisdiction, and documentation to ensure ethical and empirical deployment of interventions.

Cost/cost-effectiveness Computer-delivered interventions reduce treatment cost and barriers to treatment [56].

\section{Mindfulness-based interventions}

- Mindfulness-based interventions (MBIs) reduce alcohol, cocaine, amphetamine, marijuana, cigarette, and opioid use and craving [57]. However, these findings are limited by small sample size, lack of replicated findings, and an absence of research in adolescents.

Standard procedure Mindfulness is the development of a non-judgmental and accepting attention to the present moment [58]. Some mindfulness interventions are based exclusively on mindfulness principles (i.e., mindfulness-based stress reduction, and mindfulness-based cognitive therapy), while other interventions incorporate mindfulness with other therapeutic approaches (i.e., dialectical behavior therapy, and acceptance and commitment therapy) [59]. MBIs may be indicated for treatment of substance use because they foster a non-judgmental attitude that reduces stress, increases adaptive patterns of thinking, and increases distress tolerance. Developing the ability to tolerate negative experiences could reduce craving and relapse for individuals who use substances to avoid negative emotions, and increased awareness of low-level craving could facilitate application of skills to prevent relapse.

Contraindications

Complications

Special points

Cost/cost-effectiveness
None documented

Individuals who have impaired ability to cope with negative emotional states may have difficulty applying an intervention focusing on awareness of internal states.

MBIs have been indicated for pain management [60], so this intervention may be particularly salient for youth with co-occurring pain that may be contributing to self-medication with or abuse of prescribed pain medication or other drugs.

Undocumented

- Pediatricians and other primary care clinicians play an important role in early detection of adolescents who have initiated substance use or who may have progressed to SUD. There has been a major national initiative to enhance /increase the effectiveness of screening for adolescent substance use, misuse, abuse /dependence in pediatric and other primary care medical settings. Most states have developed and adopted SBIRT guidelines that provide primary care clinicians with screening and as- 
sessment instruments (e.g., the CRAFFT screen) [61], and assist them in brief intervention and referral to subspecialty substance treatment programs in their area.

\section{Acknowledgement}

This research was supported by T32 MH015442 and R01DA022284

\section{Compliance with Ethics Guidelines}

\section{Conflict of Interest}

Katherine A. Belendiuk owns stock or stock options in Shire Pharmaceuticals.

NIMH supports Dr. Belendiuk on a T32 MH015442 grant.

Paula Riggs has received a grant from the National Institute on Drug Abuse and is supported in employment by the University of Colorado, School of Medicine.

Human and Animal Rights and Informed Consent

This article does not contain any studies with human or animal subjects performed by any of the authors.

\section{References and Recommended Reading}

Papers of particular interest, published recently, have been highlighted as:

- Of importance

-• Of major importance

1. Merikangas KR et al. Lifetime prevalence of mental disorders in US adolescents: Results from the national comorbidity survey replication-adolescent supplement (NCS-A). J Am Acad Child Adolesc Psychiatry. 2010;49(10):980-9.

2. Johnston LD, et al. Monitoring the future national survey results on drug use, 1975-2012. Vol. I: Secondary school students. 2013, Ann Arbor, MI: Institute for Social Research, The University of Michigan

3.• Meier MH et al. Persistent cannabis users show neuropsychological decline from childhood to midlife. Proc Natl Acad Sci. 2012;109(40):E2657-64.

Prospective birth cohort study shows that adolescent-onset persistent cannabis use is associated with neuropsychological decline after controlling for education.

4. Gray KM et al. Bupropion SR and contingency management for adolescent smoking cessation. J Subst Abus Treat. 2011;40(1):77-86.

5. Gray KM et al. A double-blind randomized controlled trial of $\mathrm{N}$-acetylcysteine in cannabis-dependent adolescents. Am J Psychiatr. 2012;169(8):805-12.

6. Woody GE et al. Extended vs short-term buprenorphine-naloxone for treatment of opioid- addicted youth. JAMA: J Am Med Assoc. 2008;300(17):2003-11.

7. Riggs PD et al. A randomized controlled trial of fluoxetine and cognitive behavioral therapy in adolescents with major depression, behavior problems, and substance use disorders. Arch Pediatr Adolesc Med. 2007;161(11):1026.

8. Riggs PD et al. Randomized controlled trial of osmotic-release methylphenidate with cognitive-behavioral therapy in adolescents with attentiondeficit/hyperactivity disorder and substance use disorders. J Am Acad Child Adolesc Psychiatry. 2011;50(9):903-14.

Randomized controlled trial demonstrates that treating adolescents with ADHD with osmotic-release methylphenidate resulted in more negative urine drug screens, compared to placebo.

9. Thurstone $\mathrm{C}$ et al. Randomized, controlled trial of atomoxetine for attention-deficit/hyperactivity disorder in adolescents with substance use disorder. J Am Acad Child Adolesc Psychiatry. 2010;49(6):573-82.

10. McLellan AT et al. Drug dependence, a chronic medical illness. JAMA: J Am Med Assoc. 2000;284(13):1689-95. 
11. National Institute on Drug Abuse, Principles of Drug Addiction Treatment: A Research Based Guide 2012.

12. Hendriks V, van der Schee E, Blanken P. Matching adolescents with a cannabis use disorder to multidimensional family therapy or cognitive behavioral therapy: Treatment effect moderators in a randomized controlled trial. Drug Alcohol Depend. 2012;125(1):119-26.

13. Waldron HB, Turner CW. Evidence-based psychosocial treatments for adolescent substance abuse. J Clin Child Adolesc Psychol. 2008;37(1):238-61.

14. Tripodi SJ et al. Interventions for reducing adolescent alcohol abuse: A meta-analytic review. Arch Pediatr Adolesc Med. 2010;164(1):85.

15. Nunes EV, Levin FR. Treatment of depression in patients with alcohol or other drug dependence. JAMA: J Am Med Assoc. 2004;291(15):1887-96.

16. Liddle HA. Treating adolescent substance abuse using multidimensional family therapy, in Evidence-based psychotherapies for children and adolescents, J.R. Weisz and A. Kazdin, Editors. 2010, Guilford: New York.

17. Rigter $\mathrm{H}$, et al. Multidimensional family therapy lowers the rate of cannabis dependence in adolescents: A randomised controlled trial in Western European outpatient settings. Drug and Alcohol Dependence. 2012.

18. Hendriks V, van der Schee E, Blanken P. Treatment of adolescents with a cannabis use disorder: Main findings of a randomized controlled trial comparing multidimensional family therapy and cognitive behavioral therapy in The Netherlands. Drug Alcohol Depend. 2011;119(1):64-71.

19. Henderson CE et al. Effectiveness of multidimensional family therapy with higher severity substanceabusing adolescents: Report from two randomized controlled trials. J Consult Clin Psychol. 2010;78(6):885.

20. Robbins MS et al. Brief strategic family therapy versus treatment as usual: Results of a multisite randomized trial for substance using adolescents. J Consult Clin Psychol. 2011;79(6):713.

A randomized effectiveness trial that establishes that brief strategic family therapy is more effective than treatment as usual in reducing the median days of self-reported drug use at the end of treatment.

21. Meyers RJ, Roozen HG, Smith JE. The community reinforcement approach: An update of the evidence. Alcohol Res Health. 2011;33(4):380.

22. Slesnick $\mathrm{N}$ et al. Treatment outcome for street-living, homeless youth. Addict Behav. 2007;32(6):1237-51

23. Godley SH, Hedges K, Hunter B. Gender and racial differences in treatment process and outcome among participants in the adolescent community reinforcement approach. Psychol Addict Behav.

2011;25(1):143.
24. Dennis ML et al. The cannabis youth treatment (CYT) study: Main findings from two randomized trials. J Subst Abus Treat. 2004;27(3):197-213.

25. French MT et al. The economic cost of outpatient marijuana treatment for adolescents: findings from a multi-site field experiment. Addiction. 2002;97(s1):84-97.

26. Macgowan MJ, Engle B. Evidence for optimism: Behavior therapies and motivational interviewing in adolescent substance abuse treatment. Child Adolesc Psychiatr Clin N Am. 2010;19(3):527.

27. McHugh RK, Hearon BA, Otto MW. Cognitive-behavioral therapy for substance use disorders. Psychiatr Clin North Am. 2010;33(3):511.

28. Waldron $\mathrm{HB}$ et al. Treatment outcomes for adolescent substance abuse at 4- and 7-month assessments. J Consult Clin Psychol. 2001;69(5):802-13.

29. Winters KC, Botzet AM, Fahnhorst T. Advances in adolescent substance abuse treatment. Curr Psychiatry Rep. 2011;13(5):416-21.

30. Miller WR, Rollnick S. Motivational interviewing: Helping people change. 3rd ed. New York: Guilford Press; 2012.

31. Neighbors CJ et al. Cost-effectiveness of a motivational intervention for alcohol-involved youth in a hospital emergency department. J Stud Alcohol Drugs. 2010;71(3):384.

32. Heckman CJ, Egleston BL, Hofmann MT. Efficacy of motivational interviewing for smoking cessation: a systematic review and meta-analysis. Tob Control. 2010;19(5):410-6.

33. Hettema JE, Hendricks PS. Motivational interviewing for smoking cessation: A meta-analytic review. J Consult Clin Psychol. 2010;78(6):868.

$34 . \bullet$ Jensen CD et al. Effectiveness of motivational interviewing interventions for adolescent substance use behavior change: A meta-analytic review. J Consult Clin Psychol. 2011;79(4):433.

A meta-analytic review of 21 independent studies of 5,471 adolescents demonstrates a small but significant effect size of motivational interviewing on reducing alcohol, tobacco, and other drug use.

35. Barnett E, et al. Motivational interviewing for adolescent substance use: A review of the literature. Addictive Behaviors, 2012.

A meta-analytic review of 39 studies, 37 of which were randomized controlled trials, and 10 of which were published since 2010, examined adolescent motivational interviewing interventions for substance use and shows that two-thirds of studies improve substance use outcomes.

36. Stanger $\mathrm{C}$ et al. A randomized trial of contingency management for adolescent marijuana abuse and dependence. Drug Alcohol Depend. 2009;105(3):240-7.

37. Krishnan-Sarin $\mathrm{S}$ et al. Contingency management for smoking cessation in adolescent smokers. Exp Clin Psychopharmacol. 2006;14(3):306. 
38. Stanger C, Budney AJ. Contingency management approaches for adolescent substance use disorders. Child Adolesc Psychiatr Clin N Am. 2010;19(3):547.

39. Petry NM et al. Prize-based contingency management does not increase gambling. Drug Alcohol Depend. 2006;83(3):269-73.

40. Petry $\mathrm{N}$ et al. Give them prizes and they will come: Contingency management for treatment of alcohol dependence. J Consult Clin Psychol. 2000;68(2):250.

41. Lott DC, Jencius S. Effectiveness of very low-cost contingency management in a community adolescent treatment program. Drug Alcohol Depend. 2009;102(1):162-5.

42. Deas D, Thomas SE. An overview of controlled studies of adolescent substance abuse treatment. Am J Addict. 2001;10(2):178-89.

43. Armstrong TD, Costello EJ. Community studies on adolescent substance use, abuse, or dependence and psychiatric comorbidity. J Consult Clin Psychol. 2002;70(6):1224.

44. Lee SS et al. Prospective association of childhood attention-deficit/hyperactivity disorder (ADHD) and substance use and abuse/dependence: A meta-analytic review. Clin Psychol Rev. 2011;31:328-41.

45. Kelly JF et al. Can 12-step group participation strengthen and extend the benefits of adolescent addiction treatment? A prospective analysis. Drug Alcohol Depend. 2010;110(1):117-25.

46. Kelly JF, Urbanoski K. Youth recovery contexts: The incremental effects of 12-step attendance and involvement on adolescent outpatient outcomes. Alcohol Clin Exp Res. 2012;36(7):1219-29.

47. Godley MD, et al. A randomized trial of assertive continuing care and contingency management for adolescents with substance use disorders. Journal of Consulting and Clinical Psychology. 2013

48. Duncan AB, Velasquez SE, Nelson E-L. Using videoconferencing to provide psychological services to rural children and adolescents: A Review and Case Example. Journal of Clinical Child \& Adolescent Psychology. 2013(ahead-of-print): p. 1-13.

49. Nelson E-L, Bui T. Rural telepsychology services for children and adolescents. J Clin Psychol. 2010;66(5):490-501.
50. Marsch LA, Dallery J. Advances in the psychosocial treatment of addiction: The role of technology in the delivery of evidence-based psychosocial treatment. Psychiatr Clin North Am. 2012;35(2):481.

51. Carroll KM et al. Enduring effects of a computerassisted training program for cognitive behavioral therapy: a 6-month follow-up of CBT4CBT. Drug Alcohol Depend. 2009;100(1):178-81.

52. Schwinn TM, Schinke SP, Di Noia J. Preventing drug abuse among adolescent girls: outcome data from an internet-based intervention. Prev Sci. 2010;11(1):2432.

53. Walton MA, et al. Computer and therapist based brief interventions among cannabis-using adolescents presenting to primary care: One year outcomes. Drug and Alcohol Dependence. 2013

54. Shrier LA, et al. Real-time, contextual intervention using mobile technology to reduce marijuana use among youth: A Pilot Study. Addictive Behaviors. 2013.

55. Jarlais DCD et al. Audio-computer interviewing to measure risk behaviour for HIV among injecting drug users: A quasi-randomised trial. Lancet. 1999;353(9165):1657-61.

56. Ondersma SJ, Grekin ER, Svikis D. The potential for technology in brief interventions for substance use, and during-session prediction of computer-delivered brief intervention response. Subst Use Misuse. 2011;46(1):77-86.

57. Chiesa A, Serretti A. Are mindfulness-based interventions effective for substance use disorders? A systematic review of the evidence. Substance Use and Misuse. 2013.

58. Kabat-Zinn J. Wherever you go, there you are: Mindfulness meditation in everyday life. 1994: Hyperion.

59. Cardaciotto L. Assessing mindfulness: The development of a bi-dimensional measure of awareness and acceptance. 2005, Drexel University.

60. Chiesa A, Serretti A. Mindfulness-based interventions for chronic pain: A systematic review of the evidence. J Altern Complement Med. 2011;17(1):83-93.

61. Knight JR et al. Validity of the CRAFFT substance abuse screening test among adolescent clinic patients. Arch Pediatr Adolesc Med. 2002;156(6):607-14. 